\title{
Poétique des listes apostoliques
}

\section{Les premières énumérations d'apôtres dans la poésie latine chrétienne $\left(\mathrm{V}^{\mathrm{e}}-\mathrm{VI} \mathrm{e}^{\mathrm{e}}\right.$ siècle)}

L'histoire du christianisme, observait récemment Els Rose en introduction à un article sur le recueil des Virtutes apostolorum, fait apparaître deux grandes façons de faire référence aux apôtres : comme collège ou comme autorités individuelles ${ }^{1}$. Cette distinction essentielle joue un rôle structurant dans une récente étude consacrée aux apôtres dans la poésie et l'art chrétiens anciens : son auteur, Roald Dijkstra ${ }^{2}$, subdivise régulièrement ses chapitres en sous-parties intitulées «Apostles as a Group» et «Individual Apostles». Il y a toutefois eu également, tout au long de l'histoire chrétienne, une façon, certes moins usuelle, de se référer aux apôtres qui tient en quelque sorte le milieu entre ces deux pôles, collégial et individuel : on peut aussi se référer au groupe des Apôtres en les nommant individuellement. C'est ce que faisaient déjà les auteurs des évangiles synoptiques lorsqu'ils donnaient la liste des Douze (Mt 10.2-4 ; Mc 3.14-19; Lc 6.13-16 ; Ac 1.13), mais, au fil des siècles, ce type de référence a bien sûr trouvé bien d'autres expressions dans la littérature - on peut justement penser aux Virtutes apostolorum, qui rassemblent douze vies ou martyrs individuels - et dans l'iconographie-songer à la mosaïque de la coupole du Baptistère des Orthodoxes de Ravenne, qui figure les douze Apôtres autour d'un médaillon représentant le baptême du Christ. C'est l'appropriation par la poésie latine chrétienne de ce type de référence qui sera l'objet de cette contribution ${ }^{3}$. Je me concentrerai par conséquent sur les $\mathrm{V}^{\mathrm{e}}$ et $\mathrm{VI}^{\mathrm{e}}$ siècle, c'est-à-dire une période allant de l'apparition la première énumération versifiée des Douze aux derniers feux de la poésie d'inspiration antique dans Gaule mérovingienne, en particulier avec Venance Fortunat, qu'on qualifie à juste titre de «dernier poète de l'Antiquité» ${ }^{4}$. Je prendrai en compte à la fois son traitement littéraire et le rapport entre les poèmes étudiés et leurs sources.

Du point de vue littéraire, il importe de souligner d'emblée que la reprise poétique des énumérations d'apôtres par la poésie chrétienne ne fait que prolonger une longue tradition poétique, déjà présente dans la poésie grecque archaïque. En effet, depuis ses origines - on pense évidemment au Catalogue des Vaisseaux de l'Iliade

1 Rose (2013a) 57.

2 Dijkstra (2016).

3 La poésie latine pourrait fournir passablement d'exemples d'énumérations de quelques apôtres, mais je me concentrerai sur les énumérations ou catalogues qui à la fois montrent un intérêt pour les apôtres dans leur ensemble et indiquent leurs noms ou en tout cas un nombre significatif de ceux-ci. 4 En raison des incertitudes qui pèsent sur leur date, je ne prendrai pas en compte le petit poème $\mathrm{BHL}^{\mathrm{n}} 654 \mathrm{k}$ (Versus memoriales), bien qu'il soit envisageable qu'il appartienne encore au VI $\mathrm{e}^{\mathrm{e}}$ siècle ; je me contenterai de l'évoquer rapidement dans la conclusion. 
(II.484-780) -, listes et catalogues ont servi de matière à la poésie hellénique et, bien évidemment, il en est allé de même pour la poésie latine. La simple énumération acquerra ses lettres de noblesses avec Ovide, qu'on a pu présenter comme l'inventeur de cette forme particulière, illustrée à merveille par la longue liste d'arbres qui se mettent à couvrir une colline jusque-là nue, où Orphée joue de la lyre (Métamorphoses X.86-105) ${ }^{5}$. La poésie tardive et chrétienne tardive hérite de ce goût pour les énumérations, listes et les catalogues et assume pleinement cet héritage ${ }^{6}$. $A$ priori, donc, la liste des apôtres, figures fondatrices de l'Église, aurait pu constituer une matière poétique de choix. Néanmoins, la poésie chrétienne paraît avoir été réticente à l'intégrer : les poètes grecs n'ont montré qu'un intérêt très limité ${ }^{7}$, tandis qu'en Occident le développement sera assez lent. En la matière, la poésie latine délaissera la forme de l'énumération simple ${ }^{8}$, préférant celle de listes complexes, catalogiques, qui relient un nom à un type d'information (champ de mission, lieu de sépulture), comme le font les listes d'apôtre en prose.

La recherche des sources où les poètes puisent leurs données sur les apôtres m'amènera évidemment à étudier les rapports entre leurs compositions et les opuscules en prose que l'on désigne couramment comme listes d'apôtres. Étant donné que ces textes sont peu connus, il est sans doute utile de terminer cette introduction en les présentant très brièvement : ces petits textes, qui existent pratiquement dans toutes les langues utilisées par la chrétienté antique et médiévale, rassemblent de brèves notices sur les Douze et, le cas échéant, d'autres personnages apostoliques ou subapostoliques (Paul, Marc, Luc, certains collaborateurs de Paul, les soixante-dix disciples [cf. Lc 10.1], etc.) ${ }^{9}$. Ce genre d'opuscules a dû naître dans le courant du IV $\mathrm{I}^{\mathrm{e}}$ siècle dans le christianisme de langue grecque - comme le suggère sa diffusion rapide aussi bien en Orient qu'en Occident -, puisqu'il semble être totalement inconnu à Eusèbe $^{10}$; comme nous le verrons, le premier témoignage assuré de son existence est précisément un poème chrétien, le Natalicium 11 de Paulin de Nole, qui date de 405 environ. Le genre doit avoir connu un développement assez

5 Voir Williams (1978) 215.

6 Voir Roberts (1989) 59-61

7 Le seul poème grec contenant une énumération des apôtres qui soit certainement antérieur à la fin $\mathrm{du} \mathrm{VI}^{\mathrm{e}}$ siècle est un très bref poème de Grégoire de Nazianze (Carmina theologica I.1.19). Pour la période byzantine, il faut signaler le poème enregistré par la BHG sous le numéro 156e (on se reportera désormais à l'édition de L. G. Westerink, Michaelis Pselli poemata [Bibliotheca scriptorum Graecorum et Romanorum Teubneriana], Stutgardiae, In aedibus B. G. Teubneri, 1992, Inde Petrum et Paulum Romana461-463).

8 Celle qu'avait choisie Grégoire de Nazianze (voir n. 7).

9 Sur les listes d'apôtres, voir notamment : Dolbeau (2012), en part. VI ; Guignard (2015b); Vinogradov (2001) 121-124. Le recueil le plus complet reste l'édition, hélas très médiocre, de Schermann (1907).

10 Eusèbe n'est encore capable de donner sur les apôtres que des informations partielles ; avec lui, les données commencent seulement à être regroupées (voir Historia ecclesiastica III, 1), mais on reste loin de listes systématiques. Sur l'origine des listes d'apôtres, voir Dolbeau (2012) 178-179. 
rapide, car plusieurs listes sont datables avec un haut degré de certitude ou au moins de probabilité entre le $\mathrm{IV}^{\mathrm{e}}$ et le $\mathrm{VI}^{\mathrm{e}}$ siècle, aussi bien en latin qu'en grec.

\section{Le peu d'attrait de la poésie biblique pour l'énumération des apôtres}

Avant de s'intéresser aux énumérations que nous fournissent un certain nombre de poèmes, il convient de remarquer l'absence des noms des apôtres dans les paraphrases des évangiles ou des Actes des apôtres qui nous sont parvenues : ni Juvencus $^{11}$, ni, plus tard, Sedulius ou Arator n'ont inclus une telle liste dans leurs poèmes. Il n’y a que Sévère (de Malaga ?) à qui l'on peut laisser le bénéfice du doute, vu la conservation fragmentaire de ses De euangeliis libri XII. Pour Nonnos, le problème ne se posait pas, puisque Jean ne contient aucune liste complète des disciples de Jésus ${ }^{12}$. Quant aux centons, une telle énumération aurait évidemment été incompatible avec les lois du genre.

L'auteur de l'Hymnum de Christo (aussi désigné par son incipit : Hymnum dicat turba fratrum), en tétramètres trochaïques, qui pourrait être Hilaire de Poitiers, quoique cette paternité soit très discuté $e^{13}$, ne se soucie pas non plus de mettre en vers les noms des apôtres. Il se contente d'une référence collective à leur vocation : Duodecim uiros probauit, per quos uita discitur (v. 30). Il en nomme un seul, Judas, dont la mention sert de transition entre l'évocation des miracles de Jésus et celle de sa Passion :

Ex quis unus inuenitur, Christi Iudas traditor;

instruuntur missi ab Anna proditoris osculo (v. 31-32).

Il semble donc que l'énumération des noms des Douze, qui, en tout aux yeux de certains poètes, pouvaient présenter l'inconvénient d'être pour une bonne part des noms juifs et donc parfaitement étrangers à la tradition culturelle de la poésie grecque ou latine ${ }^{14}$, n'ait pas été, en règle générale, considérée comme une matière poétique suffisamment intéressante dans la poésie d'inspiration biblique.

11 Sur l'omission de cette liste par Juvencus, voir Dijkstra (2016) 87. Il remarque notamment : «Presumably Juvencus had omitted the names, because he wanted to avoid alienating effects in his work: he often omitted Jewish names ...». Sur la volonté de Juvencus de «déjudaïser» sa paraphrase en omettant les noms hébreux, voir aussi, par exemple, Charlet (1985) 633.

12 On trouve toutefois une liste partielle en Parphrase de Jean 21.3-8 (cf. Jn 21.2).

13 Voir les références données par Dijkstra (2016) 119 n. 206.

14 Nous pensons en particulier à l'attitude de Juvencus (voir n. 11). 


\section{Paulin de Nole}

C'est donc plutôt avec avec Paulin de Nole ${ }^{15}$ que l'enquête doit commencer. Car c'est dans son œuvre que l'on rencontre, pour la première fois dans poésie latine, une exploitation poétique d'une énumération d'apôtres. Énumération certes incomplète, car Paulin semble s'être refusé à proposer un catalogue complet ou systématique un tel catalogue n'apparaîtra que plus d'un siècle et demi plus tard, sous la plume de Venance Fortunat.

La première partie du Natalicium 11 (carmen 19 Hartel $^{16}$ ) célèbre le don qu'a fait dieux aux régions du monde en y plaçant les tombeaux des martyrs (v. 14-19) ${ }^{17}$ :

Omnis enim, quacumque manet mandatus in ora,

Martyr stella loci simul et medicina colentum est;

Namque, tenebrosum ueteri caligine mundum

Languentesque animas miseratus in orbe, Creator

Sic sacra disposuit terris monumenta piorum,

Sparsit ut astrorum nocturno lumina caelo.

Ce principe général une fois posé, Paulin va citer des exemples concrets en commençant par les apôtres (v. 54 ss.). Il passera ensuite à Cyprien (v. 141) et à d'autres martyrs d'Afrique et quelques autres martyrs d'Occident, avant d'en revenir à Nole et à Félix (v. 165), déjà évoqués au début du poème. Les apôtres occupent donc une part significative dans le catalogue des saints et martyrs de cette partie du poème.

Les apôtres et les villes et contrées abritant leurs tombeaux sont mentionnés dans deux petits ensembles de longueur inégale : trois vers sont d'abord consacrés à Pierre et Paul enterrés à Rome (v. 54-56) ; après une brève transition (v. 76-77), six vers et demi sont ensuite consacrés à neuf autres apôtres ou compagnons d'apôtres ${ }^{18}$ enterrés dans diverses cités et régions orientales (v. 78-84).

15 Pour une présentation des travaux sur Paulin publiés au cours des dernières décennies, voir Evenepoel (2016) 399-414. Sur le Natalicium XI, voir en particulier Cutino (2005) 69-79 ; Luongo (2006) 380 - 386 et 388-393. Je n’ai malheureusement pas eu accès à Luongo (2002-2006) 167-191. 16 À l'édition de W. von Hartel (Sancti Pontii Meropii Paulini Nolani carmina [Corpus scriptorum ecclesiasticorum Latinorum 30], Vindobonae, F. Tempsky, 1894), il faut préférer désormais celle de F. Dolveck, Paulini Nolani Carmina (Corpus Christianorum. Series Latina 21), Turnhout, Brepols, 2015. 17 Pour un rapide aperçu du rapport entre les martyrs et les lieux qui abritent leurs tombeaux dans la poésie latine de l'époque de Paulin, voir Fux (2003) 52-60 ; pour Paulin, on pourra consulter notamment Cattaneo (2001), 266-276.

18 Les compagnons d'Apôtres (Tite, Luc, Marc) sont regroupés à la fin (v. 83-84), ce qui suggère un statut inférieur à celui des Apôtres proprement dit, mais le fait même qu'ils soient unis à ceux-ci dans une même énumération suggère qu'ils jouissent aux yeux de Paulin d'un statut quasi-apostolique, ce que confirme le fait que, comme le remarque Dijkstra (2016) 256-257, la formule apostolicis ... corporibus des v. 335-336 s’applique manifestement à Timothée, Luc et André. 
Inde Petrum et Paulum Romana fixit in urbe,

Principibus quoniam medicis caput orbis egebat,

Multis insanum uitiis caecumque tenebris. [...]

Sic Deus et reliquis tribuens pia munera terris

Sparsit ubique loci magnas sua membra per urbes ;

Sic dedit Andream Patris Ephesoque Iohannem,

Vt simul Europam atque Asiam curaret in illis

Discuteretque graues per lumina tanta tenebras;

Parthia Matthaeum conplectitur, India Thomam,

Lebbaeum Libyes, Phryges accepere Philippum,

Creta Titum sumpsit, medicum Boeotia Lucan;

Marcus, Alexandria, tibi datus ...

Entre ces deux ensembles, une vingtaine de vers célèbre la victoire de la foi à Rome où le christianisme l'emporte désormais sur les anciens cultes païens (v. 57-75). Le second groupe de vers consacrés aux tombeaux des apôtres sera suivi d'un développement similaire, mais plus long, concernant les régions orientales (surtout l'Égypte et le monde grec ; v. 84-116), qui fait une place importante aux régions mentionnées en lien avec les apôtres aux v. 78 à $84^{19}$.

Divers thèmes et métaphores relient ces parties entre elles et avec le reste du poème. Paulin développe d'une part deux métaphores complémentaires : celle de la maladie, des soins et des médecins (les saints) et celle aveuglement et des ténèbres auxquels s'opposent l'illumination ou la guérison de la cécité. Très prégnante dans l'ensemble du poème, celle de la médecine apparaît à trois endroits clés : dans le premier ensemble de vers (principibus ... medicis, v. 55), au début du deuxième ensemble (curaret, v. 79, à propos d'André et Jean) et à la fin du même ensemble (Luc qualifié de médecin, v. $83^{20}$ ) ; elle contribue ainsi à l'unité de la section apostolique. Celle de la lumière et des ténèbres joue un rôle semblable : Paulin fait d'André et de Jean des lumières dissipant les ténèbres (discuteretque graues per lumina tanta tenebras, v. 80), ce qui fait écho aux ténèbres de la cécité évoquées dans le premier ensemble (v. 56), même si Paulin est soucieux de varier l'image. Les mêmes remarques valent également pour un autre thème, connexe, qui occupe lui aussi une place importante dans le poème : celui de la défaite et de la fuite des démons. Occupant les deux développements qui font suite à chacun des groupes de vers consacré aux apôtres, il marque à la fois à l'unité de la section et sa division en deux ensembles inégaux. Ces trois thèmes et métaphores sont étroitement liés entre eux, puisque, comme l'a relevé Michele Cutino, aussi bien les ténèbres que la maladie représentent

19 Sur la polémique antipaïenne dans cette partie du poème, voir Guttilla (2006) 355-377.

20 Paulin ne fait évidemment qu'exploiter une donnée néotestamentaire (Col 4.14), mais il semble jouer sur le double sens possible de la formule medicum Boetia Lucan [sumpsit] (v. 83). Dans le contexte, on comprend naturellement : «la Béotie a pris Luc le médecin», mais on pourrait aussi entendre «la Béotie a pris Luc pour médecin». 
l'erreur polythéiste et que Paulin, suivant en cela un topos apologétique, voit dans celle-ci l'œuvre du démon ${ }^{21}$.

La structure d'ensemble peut donc se résumer ainsi :
v. $54-56$
Pierre et Paul
v. $57-75$
défaite des cultes païens et des démons à Rome
v. $76-84$
neuf autres apôtres et compagnons d'apôtres
v. $84-140$
défaite des cultes païens et des démons en Égypte et dans le monde grec

Cette bipartition révèle la dualité fondamentale selon laquelle Paulin structure le passage : d'un côté Rome, caput orbis (v. 55), qui abrite les deux apôtres les plus prestigieux, Pierre et $\mathrm{Paul}^{22}$; de l'autre des villes ou contrées qui représentent le reste du monde (reliquis ... terris, v. 76) et se voient répartir d'autres apôtres ou personnages subapostoliques ${ }^{23}$. Cette dualité est accentuée par le fait que les deux développements sur la défaite des cultes païens construisent, au moyen d'évocations spécifiques, des univers religieux différents, typiquement romain pour le premier, gréco-oriental pour le second.

Paulin met ainsi en œuvre une dualité fondamentale entre Rome et le reste $\mathrm{du}$ monde, qui joue un rôle structurel majeur en ce qu'elle commande l'organisation de tout le passage. Elle oriente ainsi vers une lecture à la fois hiérarchique (caput orbis dotée de deux apôtres majeurs - autres villes abritant un seul apôtre ou compagnon d'apôtre) et géographique (Occident - Orient). Le lecteur est d'autant plus clairement invité à suivre ce second axe de lecture que les deux premières villes mentionnées lorsque le poète reprend l'énumération des apôtres après le développement des v. 57-75, à savoir Patras (André) et Éphèse (Jean), symbolisent explicitement l'Europe et l'Asie.

De fait, l'organisation géographique des noms n'est pas fortuite, mais dessine un parcours déterminé : après Rome, l'énumération des apôtres se dirige vers la partie orientale de l'Empire (Patras, Éphèse), puis, toujours vers l'Orient, franchit les limites de l'Empire (Parthie) pour atteindre ce qui était en quelque sorte l'ExtrêmeOrient (Inde). Il s'agit sans doute pour Paulin de montrer l'accomplissement de la

21 Voir Cutino (2005) 70. Concernant la métaphore médicale, Dijkstra (2016) 266 remarque que l'assimilation des apôtres à des médecins est une nouveauté dans la poésie latine.

22 Paulin avait déjà souligné dans d'autres poèmes le prestige que conférait à Rome la présence de leurs tombeaux ; voir Natal. 2 (= carm. 13 Hartel), v. 26-30 ; 3 (= carm. 14 Hartel), 65s (et cf. v. 85-88). Sur les liens entre les deux apôtres et Rome chez Paulin, voir Piras (2001) 277-283.

23 En fait, le principe posé aux v. 75-76 a sans doute une validité qui dépasse les seuls apôtres et s'applique également aux martyrs mentionnés à partir du v. 141. De ce point de vue, on pourrait considérer que Paulin opposerait d'une part Rome avec Pierre et Paul et d'autre part les reliquae terrae, d'abord avec le reste des apôtres, puis avec les martyrs. Il n'en reste pas moins que la section bipartite qui concerne les apôtres a sa cohérence propre, ce qui invite à faire passer la ligne de démarcation principale entre le dyptique qui concerne les apôtres (et leurs compagnons) et ce qui concerne les martyrs. 
parole par laquelle Jésus annonce à ses apôtres qu'ils seront ses témoins «jusqu'aux extrémités de la terre» (Ac 1.8). Le mouvement s’inverse alors : on revient vers l'Ouest, mais en passant par un troisième continent, l'Afrique (Libyens), pour ensuite rester dans des contrées qui évoquent le passé mythique et classique de la Grèce (Phrygie, Crète, Béotie). Enfin, le parcours, dans la section apostolique ${ }^{24}$, s'achève dans une ville qui, notamment de par son rayonnement culturel et son importance ecclésiastique en tant que siège d'un patriarcat, fait figure de pendant oriental à Rome : Alexandrie. Si, dans ce rôle, Alexandrie est préférée à Constantinople, c'est sans doute, en tout cas en partie, parce qu'en tant que ville à la fois grecque et égyptienne elle facilite la transition avec les vers qui, en décrivant la fin des cultes païens, vont évoquer la Grèce classique et l'Égypte. Néanmoins, l'absence de la Nouvelle Rome, qui aurait constitué une rivale plus sérieuse à l'Ancienne, ne paraît pas fortuite. En effet, la suite du poème montrera que Paulin est tout à fait conscient qu'elle abrite désormais des reliques apostoliques, importantes qui plus est (André et Timothée), mais son omission dans cette liste pourrait être destinée à en souligner le fait que Constantinople s'est invitée tardivement, et seulement par le biais de translations, dans le club fermé des villes abritant des tombeaux d'apôtres ${ }^{25}$.

Revenons à l'autre axe de lecture, l'axe hiérarchique : la structure bipartie du texte sert aussi à souligner l'importance de Pierre et de Paul en les séparant des autres. Cette analyse s'appuie non seulement sur la dignité particulière de Rome, caput orbis, mais aussi sur la qualification de principes medici (v. 55) accordée aux deux apôtres qu'elle abrite. La hiérarchie dicte aussi en bonne partie l'ordre des autres apôtres :

- le second bloc commence par deux autres apôtres de premier plan, André et Jean, qui font toujours partie de la première tétrade dans les listes des disciples de Jésus que donnent les évangiles. Leur dignité est soulignée par le fait qu’ils se voient attribuer trois vers, soit autant que Pierre et Paul ;

24 L'aspect géographique aura aussi son importance lorsque Paulin en viendra aux saints et martyrs postérieurs (v. 141 et suiv.), sans doute de façon complémentaire : d'Alexandrie, on passe à Carthage (toujours sur le continent Africain, mais dans sa partie occidentale) et, d'une manière générale, comme le souligne Cutino (2005) 75, «il movimento di evangelizzazione culmina infine nei santi dell'area più occidentale».

25 Voir v. 329-342. Paulin voit dans la translation des deux saints un projet divin et souligne que Constantinople tend ainsi à s'égaler à Rome (Constantinopolis, magnae caput aemula Romae, v. 338) ou presque, car il ne manque pas de souligner qu'André et Timothée (ne) sont (que) le frère de Pierre et le disciple de Paul. Il faut rappeler d'autre part que, de ce point de vue, Nole n'a apparemment rien à envier à la Nouvelle Rome, grâce aux reliques apostoliques qu'abrite la Basilica Nova (le Natal. 9 [carm. 27], 400 - 448 mentionne André, Thomas et Luc, ainsi que Jean-Baptiste et divers martyrs ; la position privilégiée de Nole en tant qu'elle abrite la tombe de Félix est aussi soulignée dans le Natal. 2 [carm. 13], 20-26, qui lui décerne même le second rang après Rome). C'est à juste titre que Cutino (2005) 79 fait remarquer que la section du Natalicium 11 consacrée aux corps des apôtres et autres saints «illustra i presupposti ideologici cui si era attenuta la costruzione della Basilica Nova, al cui interno sotto l'altare trovano posto le reliquie degli apostoli e il frammento della croce portato da Gerusalemme da Melania». 
- $\quad$ suivent quatre autres représentants des Douze : Matthieu, Thomas, Lébbée et Philippe. C'est sans doute sa dignité d'évangéliste qui vaut à Matthieu d'être cité avant Philippe (qui occupe systématiquement la cinquième place dans les listes néotestamentaires) et Thomas (qui vient toutefois après Matthieu en Mc 3, 18 et dans la plupart des manuscrits de Lc 6.15). On notera une omission importante, celle Jacques, frère de Jean ;

- les derniers nommés, Tite, Luc et Marc, ne sont plus des apôtres de plein droit, mais des compagnons d'apôtres. La mention des deux derniers permet que tous les évangélistes figurent dans la liste ${ }^{26}$.

Cet aspect hiérarchique jette un éclairage complémentaire sur le choix d'Alexandrie et de Marc pour clore l'énumération : la différence entre Rome, gratifiée de deux apôtres, et Alexandrie, qui reçoit seulement Marc, rehausse implicitement le prestige de la première en la plaçant plus non seulement que les autres villes, mais même qu'un autre siège patriarcal.

Ce parallèle structurant entre deux centres culturels et religieux est l'une des explications du nombre impair des personnages retenus, puisque, pour finir avec une ville qui fasse pendant à Rome, Paulin devait impérativement terminer avec une seule ville (et un seul apôtre, Rome étant la seule à pouvoir en revendiquer deux). Cette imparité est cependant plus qu'une nécessité d'organisation : le fait de citer onze personnages, et non douze, pour égaler au moins symboliquement le nombre des apôtres ${ }^{27}$, souligne le caractère incomplet, anthologique, de l'énumération. Il montre que Paulin n'entend nullement donner une liste exhaustive, comme le suggère d'ailleurs le sic qui ouvre le second groupe de vers (v. 78) : il fait apparaître les apôtres cités comme de simples illustrations de la répartition des tombeaux des saints dans le monde. Paulin s'est contenté d'un choix subtil, qui permet de concilier ordonnancement hiérarchique et géographique. J'ajoute que l'agencement même de l'énumération en souligne le caractère impair et donc incomplet : jusqu'à Marc, les apôtres et leurs compagnons sont toujours groupés deux à deux, avec un seul verbe pour exprimer leur lien avec leur lieu de sépulture. Paulin veille à varier son énumération en employant des verbes différents ${ }^{28}$ et diversifiant les références géographiques : adjectif (Romana, v. 54), noms de ville (Patras, Éphèse), nom de contrées (Parthie, Inde), noms de peuples (Libyens, Phrygiens), noms de régions (Crète,

26 L'association des apôtres et des évangélistes dans le poème est peut-être à rapprocher de leur association dans la mosaïque qui décorait l'abside de la Basilica Nova, récemment construite (400 403). Elle représentait en effet la Trinité, les apôtres et les évangélistes ; voir Trout (1999) 151.

27 Un peu plus d'un siècle plus tard, les douze noms inscrits sur le mausolée de Théodoric à Ravenne (texte chez Bovini (1977) 83) donnent un exemple d'une liste de douze noms qui intègre Paul, les évangélistes Marc et Luc et omet par conséquent trois des Douze (Barthélemy, Jacques fils d'Alphée, Thaddée-Lebbée ou Jude).

28 Pierre et Paul : fixit (v. 54) ; André et Jean : dedit (v. 78) ; Matthieu et Thomas : complectitur (v. 81); Lébbée et Philipppe : accepere (v. 82) ; Tite et Luc : sumpsit (v. 83). 
Béotie). Au terme de l'énumération, la référence à Marc joue sur des reprises (nouveau nom de ville, premier et unique réemploi d'un verbe ${ }^{29}$ ), mais aussi et surtout sur des ruptures : le passage à une formulation passive avec apostrophe à la ville (Marcus, Alexandria, tibi datus, v. 84), qui tranche nettement avec les constructions employées jusque-là ${ }^{30}$, et surtout, variatio ultime, la mention isolée d'un nom, qui rompt avec le rythme de deux noms par vers auquel Paulin avait habitué l'auditeur. Ces analogies et différences accentuent l'effet de clôture (aussi bien par rapport à l'ensemble de l'énumération, depuis Pierre et Paul, que par rapport à sa seconde partie, depuis André et Jean), mais elles soulignent aussi l'isolement de Marc et, par là, l'incomplétude de la liste.

En montrant que la liste constituée par Paulin est délibérément incomplète anthologique, pourrait-on dire -, l'analyse littéraire du passage amène un résultat important du point de vue de la Quellenforschung : si le poète n'indique pas les lieux de sépulture de l'ensemble des apôtres, ce n'est pas nécessairement qu'il serait dans l'incapacité de fournir une liste complète, faute d'information sur certains d'entre eux ; il semble s'agir d'un choix délibéré, qui sélectionne des données en fonction d'un dessein complexe et précis.

D’une manière générale, la poésie de Paulin ne manifeste que peu d'intérêt pour les figures apostoliques secondaires ${ }^{31}$, si bien que ce passage fait figure d'exception dans son œuvre. Ces vers sont les seuls dans lesquels Paulin mentionne certains des Douze. Si l'on remarque que l'ensemble des données qu'ils fournissent portent tous sur un seul et même point, à savoir la localisation du tombeau des Apôtres, cette exception n'est pas à expliquer par une connaissance superficielle des traditions apocryphes concernant certaines figures apostoliques ${ }^{32}$, mais bien par l'usage d'une liste d'apôtres, plus précisément d'une liste indiquant leurs lieux de sépulture. Cette hypothèse est confirmée par les convergences remarquables que l'on constate entre

29 Datus (v. 84) renvoie à dedit, employé au début de la seconde partie de l'énumération (v. 78) pour André et Jean.

30 Tous les autres noms étaient à l'accusatif, le sujet étant soit Dieu qui attribue des apôtres à une ville (Pierre et Paul, v. 54 ; André et Jean, v. 78), soit une région qui les prend ou reçoit (les six noms mentionnés aux v. 81-83).

31 Dijkstra remarque que, vu l'ampleur de l'œuvre de Paulin, on s'attendrait à ce que les Apôtres soient nommés plus souvent qu'ils ne le sont. De fait, l'intérêt du poète s'avère sélectif : il mentionne surtout les principaux, notamment Pierre et Paul, une partie des occurrences étant liées à leur production littéraire (Paul) ou à la présence de leurs reliques à Nole (André et Thomas). Certains ne sont cités que dans notre poème (Matthieu, Philippe, [Thaddée-]Lébbée), tandis que cinq d'entre eux ne sont même jamais mentionnés dans ses écrits (les deux Jacques, Barthélemy, Simon et Matthias), sans compter Judas (mentionné uniquement dans des lettres) ; voir Dijkstra 2016, p. 243s. On peut ajouter que, dans son œuvre poétique, Marc (v. 84), Tite (v. 83) et Timothée (v. 337.341-342) n'apparaissent que dans notre poème (Marc et Timothée étant mentionnés respectivement une et trois fois dans les lettres de Paulin, l'un en tant qu'évangéliste et l'autre en lien avec les épîtres qui lui sont adressées), tandis que Luc ne se retrouve que dans le Natal. 9 (= carm. 27 Hartel, v. 424s.). Dans ces conditions, la mention de Lébbée, de Philippe ou encore de Tite dans le Natal. 11 est remarquable. 32 Voir Dijkstra (2016) p. 266. 
les données reproduites par Paulin et celles que fournissent diverses listes anciennes apparentées entre elles, qui sont conservées en grec, en latin et en syriaque ${ }^{33}$. Je ne m'étendrai pas sur ce point ici, mais il faut relever l'apport mutuel entre ces listes et le poème : celles-là nous donnent à voir à quoi ressemblait la source de Paulin, tandis que celui-ci atteste que l'ancêtre commun de ces listes existait déjà en 405 .

Prose particulièrement sèche, les listes d'apôtres représentent à première vue la plus improbable des sources pour un poète. En y puisant son inspiration pour ce passage, Paulin pose un geste qui aura des répercussions importantes dans la tradition poétique postérieure de l'Occident. Il fait entrer la forme de la liste d'apôtres dans le domaine de la poésie chrétienne. Il le fait toutefois selon des modalités particulières : il ne l'y introduit pas sous la forme d'une simple simple énumération des noms des apôtres - qui n'a jamais «pris» dans la tradition poétique tardoantique -, mais en tant que liste «à double entrée» qui relie un apôtre et un type précis d'information ${ }^{34}$. Paulin n'aura certes pas d'imitateur immédiat, mais, comme je le montrerai, lorsque Venance Fortunat reprend et systématise ce type de liste et confirme pour les siècles à venir son droit de cité en poésie, il paraît se souvenir de ces vers de Paulin.

\section{Venance Fortunat}

L'œuvre de Venance Fortunat ${ }^{35}$ témoigne d'un intérêt inégalé pour les énumérations d'apôtres, puisqu'elle en compte deux ou trois, selon qu'on considère ou non l'In laudem sanctae Mariae comme étant, ou non, une œuvre authentique. La question n'est sans doute pas définitivement tranchée, mais, étant donné que l'hypothèse d'un imitateur paraît plus probable ${ }^{36}$, je traiterai ce poème à part. Quant aux deux

33 Je reviendrai sur ce thème dans un autre travail. Qu'il suffise ici de renvoyer à la liste syriaque éditée par Sims-Williams (1994) et à la liste latine dite Doopa (éd. Dolbeau (2012), II) dont une des sources semble proche de celle de la liste syriaque ; il faudrait ajouter une liste grecque inédite dont la BHG classe certains témoins sous BHG 156c (à tort, selon moi).

34 Cette appropriation poétique pourrait avoir été favorisée par l'association opérée par Prudence entre des martyrs et leur lieu de sépulture dans l'hymne 4 du Peristephanon (v. 15-52), si ce poème s'avérait être antérieur à celui de Paulin (sur cette question, voir Trout (1999) 102 et n. 133).

35 Sur Venance Fortunat, voir en particulier Roberts (2009).

36 Pour F. Leo, le caractère apocryphe de cette pièce était évident en raison du style et de la métrique (MGH. Auct. ant. 4, 1, p. XXIV). Son authenticité a cependant été défendue par Blomgren (1934) (non uidi). Dans son édition, Reydellet (2004) 165 n. 1 fait valoir la présence de ces vers parmi les œuvres authentiques de Fortunat dans plusieurs manuscrits ainsi que les rapprochements avec VIII.3, qu'il interprète comme un signe d'authenticité. La proximité en matière de christologie avec la lettre de remerciement à l'empereur Justin pour l'envoi des reliques de la Sainte-Croix l'amène à suggérer qu'«il pourrait s'agir ... d'une composition datant des débuts de Fortunat au moment de l'arrivée de la relique à Sainte-Croix (App. 2)». En revanche, dans un récent article où il se livre à une analyse attentive des particularités métriques, Ceccarelli (2015) 102, incline en faveur de l'attribution à un imitateur de Fortunat, sans toutefois prétendre formuler des conclusions définitives : «L'ipotesi che 
poèmes d'authenticité indiscutable, V.2 et VIII.3, bien qu'ils soient sont plus ou moins contemporains ${ }^{37}$, ils traitent le thème apostolique de façon différente.

\title{
Le Carmen V.2
}

Le Carmen V.2, rédigé au nom de Radegonde et des moniales de la Sainte-Croix de Poitiers, est adressé à Martin de Braga, évêque de Galice, ce qui nous place avant la mort de celui-ci, survenue en $580^{38}$. Les premiers mots, lumen apostolicum - une image qui n'est pas sans évoquer le Natalicium 11 de Paulin - introduisent le thème du ministère et de la prédication apostolique. Suit une énumération de sept saints six apôtres, puis Martin de Tours -, pour mieux célébrer cet autre Martin :

\author{
Lumen apostolicum cum spargeret una Triades \\ exciperetque nouum mundus honore diem \\ ut tenebras animae lux sementiua fugaret \\ et claram hauriret mens oculata fidem, \\ redditur auulsis spinis urbs Romula princeps, \\ principis egregii uomere culta Petri. \\ Paulus ad Illyricos Scythicas penetrando pruinas \\ dogmate feruenti frigora soluit humi; \\ Mattheus Aethiopos adtemperat ore uapores \\ uiuaque in exusto flumina fundit agro. \\ Bellica Persidis ${ }^{39}$ Thomae subiecta uigori \\ fortior efficitur uicta tiara Deo. \\ Lurida perspicuo datur India Bartholomeo ${ }^{40}$. \\ Andreae monitis extat Achaia seges. \\ Ne morer adcelerans, Martini Gallia prisci \\ excellente fide luminis arma capit. \\ Martino seruata nouo, Gallicia, plaude:
}

\footnotetext{
mi sembra spiegare meglio la situazione è quella di un imitatore sufficientemente abile per avvicinarsi alla tecnica di Venanzio, ma non abbastanza abile (o non abbastanza interessato) per non staccarsene in alcuni tratti caratterizzanti».

37 Voir Reydellet (1998) 14 n. 21.

38 Voir Labande-Mailfert (1986) 47.

39 Persidis est volontiers considéré comme un nominatif (voir à ce propos Nisard (1887) 145 n. 1 sur V.2), opinion qui peut s'appuyer sur un parallèle dans le De excidio Thoringiae (bellica Persidis seu te Byzantion optat, v. 95) ; on rappellera d'ailleurs que, si Fortunat n'emploie jamais Persis ni Persia, il invente en VIII.149, un nominatif plus étrange encore : Persida. Néanmoins, dans ces vers, j'inclinerais à rattacher bellica à tiara en faisant de Persidis un génitif.

40 L'adjectif luridus paraît désigner ici une couleur foncée (cf. TLL VII, 2 [1978], p. 1861, 1. 12-16). Quant à perspicuo, Reydellet (1998) 14 n. 22, y voit une allusion à la «transparence d'âme» dont témoigne Jn 1, 47, tandis que Spoth le prend au sens de «insignis, clarus» (TLL X, 1, p. 1746, 1. 55.73s.). Faudrait-il ici prendre l'adjectif au sens actif (cf. TLL X, 1, p. 1749, 1. 27-44), l'idée étant que Barthélemy sera capable de jeter sur les Indiens un regard (spirituel) que la couleur foncée de leur peau ne saurait arrêter?
} 
sortis apostolicae uir tuus iste fuit.

qui uirtute Petrum praebet tibi, dogmate Paulum,

hinc Iacobi tribuens, inde Iohannis opem (v. 1-20).

Alors que le jeu sur l'homonymie (v. 17) fait du destinataire l'égal de saint Martin, le lien établi avec les apôtres rehausse encore son prestige et ce, doublement. D’une part, ses qualités sont comparées à celles de quatre apôtres éminents : Pierre, Paul, Jacques (le Majeur) et Jean (v. 19-20). D'autre part, Fortunat met en œuvre un procédé particulièrement intéressant dans la perspective de cette étude : il insère en quelque sorte Martin de Galice, élevé au rang d'apôtre (v. 18.44 $4^{41}$ ), dans une répartition géographique des apôtres.

Je reviendrai plus tard sur le problème des sources de Fortunat et je me concentrerai pour l'instant sur l'organisation de la matière. Il apparaît très vite que les six apôtres retenus le sont surtout en fonction d'une logique géographique. À tout seigneur, tout honneur : Fortunat commence par Pierre, associé à Rome. Il ne pouvait en aller autrement et la qualification de princeps accordée à la fois à la ville et à son apôtre, effet encore accentué par le choc des deux formes de l'adjectif (urbs Romula princeps || principis ... Petri, v. 5-6), le souligne fortement. Comme lorsque Paulin énumère les apôtres et comme dans un certain nombre de listes en prose, en vertu du lien étroit existant entre les deux apôtres, Paul vient immédiatement après Pierre. Néanmoins, Fortunat ne fait pas référence à Paul en lien avec Rome, mais lui assigne une tout autre fonction dans sa géographie apostolique : il évoque sa mission à destination de l'Illyrie en le faisant passer par «les frimas de la Scythie». La référence à l'Illyrie évoque la limite atteinte par Paul dans sa mission selon $\mathrm{Rm} 15.19$ (... ita ut ab Hierusalem per circuitum usque in Illyricum repleuerim evangelium Christi) ; elle peut aussi se trouver dans la section consacrée à Paul dans les Virtutes apostolorum $^{42}$, qui, comme on le verra, ont servi de source à Fortunat. Plus surprenante (et sans équivalent dans de cette source) est l'allusion à la Scythie, contrée plus ordinairement associée à André ${ }^{43}$ : à ma connaissance, aucun texte n'y envoie saint Paul. De quelque manière qu'il faille expliquer ou interpréter cette donnée ${ }^{44}$, le but de l'opération est évident : présenter Paul (v. 7s.) comme l'apôtre des régions froides du Nord pour en faire le pendant de Matthieu (v. 9s.), qui évangélise les régions chaudes du Sud, symbolisées par l'Éthiopie. Avec les deux apôtres suivants,

41 Les v. 17-18 sont répétés à l'identique aux v. 43-44.

42 Ch. 7 (Fabricius, Codex apocryphus, $1719^{2}$, pars 2, p. 452).

43 Voir en particulier Eusèbe, Histoire ecclésiastique III.1.1 ; la Scythie fait aussi partie des régions évangélisées par André dans de nombreuses listes d'apôtres, à commencer par l'Anonyme I (voir Guignard (2015a) 187 ; Schermann (1907) passim).

44 Peut-être faut-il interpréter les Scythicae pruinae de façon métaphorique, le poète voulant alors suggérer qu'en se rendant jusqu'en Illyrie l'Apôtre serait entré dans les frimas septentrionaux. Néanmoins, dans un texte où les références géographiques n'ont d'ordinaire rien de métaphorique, il n'est pas évident que telle ait été son intention. Je serai amené plus loin à explorer une autre piste : une confusion avec une indication relative à André (voir p. 381). 
le regard se tourne vers l'Est, d'abord avec la Perse (Thomas, v. 11s.), puis l'Inde (Barthélemy, v. 13), qui représente l'Extrême-Orient. Après le Nord, le Sud et l'Est, le lecteur attendrait l'Ouest, mais Fortunat insère André (v. $14^{45}$ ), probablement pour faire pendant à son frère Pierre, qui avaient ouvert l'énumération ; sans doute s'agitil d'incarner, en face de Rome, le pôle grec de la chrétienté, symbolisé par l'Achaïe. Cette escale inattendue à Patras amorce néanmoins le mouvement vers l'Occident avec un dernier apôtre, mais c'est avec d'autres saints qu'il s'achèvera, en deux étapes progressives, de façon tout à fait parallèle à ce qu'on a constaté pour le mouvement vers l'Orient (Perse, puis Inde) : la Gaule apparaît d'abord, associée à Martin de Tours (v. 15s.), puis la Galice, où s'exerce l'apostolat du «nouveau Martin» (v. 17s.). De la sorte, Fortunat relie habilement son pays d'adoption et celui de son destinataire, tandis que le passage par Martin de Tours comme apôtre de la Gaule s'avère indispensable pour situer l'apostolat de son homonyme dans le prolongement de celui des Apôtres.

Le passage des Apôtres à Martin de Tours s'accompagne de la formule ne morer adcelerans. Elle a manifestement pour fonction de justifier le passage à ce saint «moderne» en omettant la façon dont la lumière de l'Évangile s'est répandue entre l'âge apostolique et celui-ci. Cependant, plus qu'à l'histoire générale de l'évangélisation du monde, les références apostoliques de tout le passage (dès le lumen apostolicum du v. 1) suggèrent que Fortunat pense à la mission des autres apôtres. Il est donc vraisemblable que, comme Paulin, Fortunat s'inspire dans ces vers du fonctionnement d'une liste d'apôtres. Le caractère très partiel de l'énumération - six apôtres, correspondant symboliquement à la moitié du collège apostolique ${ }^{46}$ - est manifestement lié à la géographie symbolique mise en œuvre : seuls un apôtre pour le Nord, un pour le Sud et deux pour l'Orient (pour faire pendant aux deux Martins) étaient absolument nécessaires à Fortunat, qui toutefois ne pouvait guère omettre Pierre et a ajouté André par souci de symétrie. Comme Paulin avant lui, Fortunat exploite le potentiel poétique des listes d'apôtres, mais sans se sentir tenu à produire une énumération complète. Cependant, alors que son prédécesseurs s’intéressait aux tombeaux des apôtres, ces vers de Fortunat reflètent un autre intérêt des listes en prose : les champs de mission.

\footnotetext{
45 Je comprends le v. 14 différemment que ne l’a fait Reydellet («L’Achaïe devient le champ des prédications d'André») : «Grâce aux prédications d'André, l'Achaïe devient un champ ensemencé (ou : une moisson)» (cf. l'expression euangelicam segetem satam caelesti semine employée par Pierre Chrysologue à propos de la parabole du Bon Grain et de l'Ivraie [Sermon 96, 1. 61, éd. Olivar, CCSL $24 \mathrm{~A}])$.

46 Symboliquement seulement, car l'adjonction de Paul suppose un total de treize apôtres.
} 


\section{Le Carmen VIII.3 (De uirginitate) ${ }^{47}$}

Composé à l'occasion de la consécration d'Agnès de Poitiers comme abbesse de la Sainte-Croix (peu avant 576) ${ }^{48}$, le De uirginitate (Carm. VIII.3) constitue un «épithalame mystique ${ }^{49}$ », qui reprend en le christianisant le thème du cortège divin ${ }^{50}$. Dans la partie initiale du poème (v. 1-46), qui décrit le cortège céleste célébrant le Créateur, le groupe des apôtres apparaît entre les saints de l'Ancien Testament et les martyrs, qui précèdent la Vierge Marie et des vierges. À ce stade, cependant, concernant les trois premiers groupes (v. 11-24), l'évocation se limite à quelques représentants éminents : Abraham et Moïse, Pierre et Étienne, respectivement.

Ce n'est que plus loin, après avoir célébré l'union particulière du Christ et des vierges (v. 97-128), que Fortunat reprendra de façon plus détaillée le thème apostolique en mentionnant douze apôtres, ainsi que Marc et Luc. Le chœur des «dignitaires du ciel» (siderei proceres, v. 129) convoqués aux noces du Roi comprend, outre les anges, les vingt-quatre anciens-prophètes (bis duodena senum ... uatum ${ }^{51}$; Fortunat semble amalgamer les seniores d'Ap 4, 4 et les prophètes de l'Ancien Testament ${ }^{52}$ ), Élie et Hénoch sur leurs chars, la Vierge Marie, Paul et Pierre, étroitement associés, André et sept autres apôtres Marc, Luc, Cyprien, et d'autres martyrs, presque tous d'Europe occidentale (v. 129-186). Parmi ce chœur des saints, les apôtres et les martyrs sont systématiquement associés à un lieu, qui est celui de leur sépulture, bien que cela reste souvent implicite. Cette association s'exprime selon deux modalités différentes : soit le saint vient de l'endroit en question (c'est le cas de Paul et Pierre, v. 138-140, ou de Victor, v. 155, etc.) ; soit, le plus souvent, la ville envoie le saint (c'est le cas de tous les autres apôtres, de Cyprien, v. 153, etc.) ${ }^{53}$.

47 La partie du poème qui concerne les apôtres (v. 137-152) s'est vu attribuer une entrée dans le Novum supplementum de la BHL (654ke), d'après Schermann (1907) 215s. Cette entrée ne se justifie guère, si ce n'est peut-être parce que ce passage a circulé sous forme d'extrait, en tout cas dans le Montepesulanus 39 (XII siècle), mais avec un incipit différent de celui qu'indique le Novum supplementum. Si je comprends bien les indications données par l'apparat de Schermann, l'extrait, pourvu d'une brève indication sur son auteur et sa provenance, commence en fait avec les v. 129s. et saute ensuite au v. 137. Ce texte présente une variante intéressante : le v. 144 par lequel Fortunat expédiait le sort des deux Jacques a été remplacé par trois vers qui reflètent, pour Jacques le Majeur, la tradition qui l'associe à la Galice (Alphaei Iacobum Ierusalem tribuit || Quam repetunt populi Iacobum natum Zebedaei || Galiciae tellus mittit ad astra poli). Cette adaptation peut difficilement être antérieure au IX ${ }^{\mathrm{e}}$ siècle (sur la légende espagnole, voir Starowieyski (1996) 198-201 ; Burnet (2014) 331-338). 48 Voir Reydellet (1998) 129 n. 10. Sur Agnès, fille spirituelle de Radegonde, voir par ex. Joye (2008). 49 Selon l'expression de Campanale (1980) 75-128, article que je n'ai malheureusement pas pu consulter.

50 Voir Guérard (2011) 10-15.

51 Cf. Carmen X.7.16.

52 Ce rapprochement a peut-être été facilité par des exégèses assimilant les vingt-quatre vieillards aux livres de l'Ancien Testament ; cf. Victorin de Pettau, Commentaire de l'Apocalypse 4.4.

53 Roberts (2009) 173s., observe : «There is an ambiguity ... whether the primary actors are the saints of the personified cities. A wedding celebration in heaven fades into or is combined with a ceremony 
Cette scène a été rapprochée du cortège des provinces dans le Panégyrique de Majorien (Carm. 5.40 - 53) de Sidoine Apollinaire et, surtout, de l'Hymne en l'honneur des dix-huit saints martyrs de Saragosse (Peristephanon 4) de Prudence, où l'on trouvait un procédé de personnification des cités qui envoient les martyrs qu'elles abritent très proche de celui que Fortunat met en œuvre ${ }^{54}$. Cette observation plaide fortement en faveur d'une imitation de cet hymne dans le Carmen VIII, 3 et ce dernier rapprochement peut en outre s'appuyer sur le fait que l'énumération des martyrs commence par Cyprien aussi bien chez Fortunat (v. 153) que chez Prudence (v. 17). Il me semble toutefois qu'à côté du poème de ce dernier, Fortunat se souvient du Natalicium 11 de Paulin, où l'énumération des martyrs commence également par l'évêque de Carthage (v. 123). En témoignent à la fois des analogies structurelles que quelques coïncidences dans l'expression. Certes, l'énumération de Fortunat est plus compacte que celle de Paulin, dont les divers ensembles sont, comme je l'ai noté, entrecoupés par de longs développements, mais on retrouve une séquence commençant par Pierre et Paul, étroitement associés, suivis par André et Jean, d'autres apôtres et évangélistes (Marc et Luc, quoique dans l'ordre inverse), des martyrs, dont l'énumération commence par Cyprien ${ }^{55}$ et comprend aussi Vincent (de Saragosse) ${ }^{56}$ et Martin de Tours ${ }^{57}$. La présence de Marc et Luc mérite d'être relevée, car ils ne figurent pas dans la source à laquelle Fortunat emprunte ses localisations apostoliques (les Virtutes apostolorum, comme on le verra), mais sont mentionnés par Paulin. À ces analogies structurelles s'ajoutent quelques similitudes dans la formulation. Le terme proceres (v. 129) était certes passablement banalisés8 mais n'en est pas moins un des termes clés du poème de Paulin (cf. v. 10), où il apparaît, comme chez Fortunat, au début de l'énumération des saints (v. 51), mais il faut signaler qu'il apparaît aussi dans le poème de Prudence (v. 191). Bien que cela n'ait rien d'original, je relève que Rome est qualifiée de caput orbis par Fortunat (v. 140) aussi bien que par Paulin (v. 55). Enfin, plus significatif paraissent les échos entre les vers où Paulin affirme que Dieu a donné André à Patras et Jean à Éphèse

Vt simul Europam atque Asiam curaret in illis

Discuteretque graues per lumina tanta tenebras (v. 79s.)

of consensus in the court of the heavenly king, with each city presenting its most precious possession with which it is most closely identified, its saint.»

54 Voir Roberts (2009) 174 ; Guérard (2011) 13-15. Roberts (2009) 174s. estime que ces représentations poétiques ont peut-être été influencées par des représentations de scènes similaires dans l'art tardo-antique.

55 Le rapport entre Paulin et Prudence sur ce point n'est sans doute pas fortuit, mais la question du rapport entre leurs poèmes respectifs dépasserait le cadre de cette recherche.

56 Vicentius exstat Hiberis (Paulin, v. 153) ; Vincenti Hispana surgit ab arce decus (Fortunat, v. 154)

57 Gallia Martinum (sumpsit) (Paulin, v. 154) ; Martinum Gallia mittit (Fortunat, v. 163)

58 Sur l'appropriation de ce terme par les poètes latins chrétiens (notamment Paulin), voir Doignon (1961) 152-156. 
et ce vers que Fortunat place à la suite de l'énumération des martyrs :

Europae atque Asiae quis lumina tanta recurrat? (v. 173).

Fortunat fait ainsi écho à une métaphore que Paulin utilise de façon récurrente dans son poème. L'énumération des apôtres et des saints du Carmen VIII.3 semble donc s'inspirer de deux modèles : d'une part, l'hymne de Prudence, dont il reprend en particulier l'image des villes qui présentent leurs martyrs (alors que chez Paulin l'idée est exactement inverse : les martyrs sont des dons que Dieu fait aux villes qui abritent leurs tombeaux) ; d'autre part, le poème de Paulin, dont il imite en particulier la structure.

Comme dans le poème V.2, Fortunat développe la poétique de l'énumération des apôtres, en lien, cette fois, avec leurs lieux d'ensevelissement. Il la déploie en suivant un schéma géographique qui va globalement de Rome (Pierre et Paul) vers l'Orient romain (André, Jean, Philippe), puis des contrées plus lointaines (Thomas, Barthélemy, Matthieu, Simon et Jude), mais qui n’a pas l'aspect contraignant de celui de V.2. Le poète est donc libre de donner une énumération complète des Douze, auxquels il ajoute en outre les deux évangélistes qui ne sont pas de leur nombre. C’est, en tout cas à notre connaissance, une première dans la poésie latine.

\section{Les sources des données de Fortunat concernant les champs de mission et les tombeaux des apôtres}

Il reste à revenir sur la question des sources de Fortunat. L'attention de la critique s'est surtout concentrée jusqu'ici sur VIII.3. On a depuis longtemps relevé l'accord des données de ce poème avec celles des Virtutes apostolorum (qui furent longtemps connues comme "Collection du Pseudo-Abdias» et le restent parfois ${ }^{59}$ et qui sont parfois désignées comme Actes latins des apôtres) : accord substantiel en ce qui concerne l'ordre des apôtres, complet en ce qui concerne les données géographiques $^{60}$ :

59 Sur cet ensemble (passablement mouvant) de passions, voir en particulier les travaux de Rose (2012) 11-45 ; Rose (2013a) 57-96 ; Rose (2013b) 227-268 ; voir également Alibert (2005) 735-864. 60 Dans le tableau ci-dessous, je suis l'ordre du poème VIII, 3 et j'indique par des numéros les sections des Virututes dans la colonne qui leur est consacrée (indications fondées sur Rose (2013a) 60 ; la réalité des manuscrits est cependant plus complexe, cf. Rose (2012) notamment 32). Quant au Carmen V.2, les apôtres y apparaissent dans l'ordre suivant : (1) Pierre, (2) Paul, (3) Matthieu, (4) Thomas, (5) Barthélemy, (6) André ; je ne prends pas en compte Jacques et Jean, qui apparaissent un peu plus loin (v. 20), dans un autre contexte. 


\begin{tabular}{|c|c|c|c|}
\hline & Carm. V.2 (mission) & Carm. VIII.3 (tomb.) & Virtutes apostolorum \\
\hline Paulus & $\begin{array}{l}\text { ad Illyricos Scythicas penetrando } \\
\text { pruinas }\end{array}$ & \multirow{2}{*}{$\begin{array}{l}\text { Romana ... } \\
\text { - ab arce }\end{array}$} & [1] Rome \\
\hline Petrus & urbs Romula & & [2] Rome \\
\hline Andreas & Achaia & Achaia & [3] Patras \\
\hline Iohannes & & Ephesus & [5] Éphèse \\
\hline \multirow{2}{*}{ [les deux] Jacobi } & & \multirow{2}{*}{ terra beata } & [4] Jérusalem \\
\hline & & & [6] Jérusalem \\
\hline Philippus & & Hierapolis & [7] Hiérapolis \\
\hline Thomas & Persidis $^{61}$ & Edessa & [8] Inde (m.) / Édesse (s.) \\
\hline Bartholomaeus & India & India & [9] Troisième Inde \\
\hline Mattheus & Aethiopos & Naddauer & $\begin{array}{l}\text { [10] Naddaver } \\
\text { (Éthiopie) }\end{array}$ \\
\hline Simon & & Persida $^{62}$ & \multirow{2}{*}{$\begin{array}{l}{[11-12] \text { Suanir }} \\
\text { (Perse) }\end{array}$} \\
\hline ludas & & - & \\
\hline Marcus & & Aegyptus & \\
\hline Lucas & & pas de lieu indiqué & \\
\hline
\end{tabular}

Concernant Marc et Luc, il est intéressant de relever que des passions de ces deux évangélistes circulent souvent aux marges des Virtutes $^{63}$, si bien que Fortunat pourrait s'inspirer à leur propos d'un recueil qui incluait leurs passions ou peut-être seulement celle de Marc. Le poème de Paulin est une autre source possible concernant Marc et Luc. Il faut cependant observer que le lien entre Marc et l'Égypte est une donnée des plus répandues et que Fortunat ne donne pas d'indication géographique à propos de Luc, si bien que leur mention ne dépend pas forcément d'une source précise.

Quoiqu'il en soit, la convergence entre Fortunat et les Virtutes est impressionnante. Lipsius, suivi par Duchesne, en concluait avec assurance que le poète avait connu et utilisé cette collection ${ }^{64}$. De fait, l'étendue de l'accord ne laisse guère de doutes sur le fait que Fortunat s'est servi la série de vies et passions qui constituent les Virtutes apostolorum, ce qui implique qu'elles circulaient déjà en Gaule à la fin du

\footnotetext{
61 Voir n. 39.

62 Voir n. 39.

63 Voir Rose (2013a) 60.

64 Voir Lipsius (1883-1890), I, 165-167 ; Duchesne (1895) 73.
} 
$\mathrm{VI}^{\mathrm{e}}$ siècle. Malgré la prudence d'Els Rose ${ }^{65}$, cela paraît d'autant moins douteux que Fortunat rejoint les Virtutes sur des points précis et des traditions rares, voire uniques : association de Simon et de Jude, Barthélemy en Inde, Matthieu dans la ville éthiopienne de Naddaver ${ }^{66}$ (donnée qui n'apparaît dans aucune autre source susceptible de remonter à l'époque de Fortunat). Ces accords, soulignés par Lipsius, ne sont pas contestés par E. Rose, qui admet que les Virtutes sont la seule source connue correspondant en tout au poème de Fortunat. Elle fait néanmoins valoir qu'il est également possible que le poète utilise une source inconnue comparable au Breviarium apostolorum ou à la Notitia des locis sanctorum apostolorum, dont elle est proche ${ }^{67}$. Ce n'est pas exclu, mais, à moins d'admettre un singulier hasard, il faut supposer qu'une telle liste dépendrait des Virtutes ${ }^{68}$. À mes yeux une hypothèse plus économique pour expliquer la récurrence dans deux poèmes des mêmes données serait que Fortunat ait fait pour son usage personnel un relevé des indications géographiques que les Virtutes fournissent pour chaque apôtre.

De fait, le Carmen V.2 reflète pour l'essentiel les mêmes données que VIII, 3, au moins au sens où la ville indiquée dans ce dernier poème appartient à la contrée indiquée dans le premier, comme on le constate dans le cas de Naddaver. Il faut aussi tenir compte de la différence de point de vue entre ces deux poèmes : l'un s'intéresse aux champs de mission des apôtres, l'autre les relie aux villes qui abritent leurs tombeaux ; le cas de Paul illustre bien cette différence.

Les Virtutes doivent donc avoir ici aussi servi de source ${ }^{69}$, mais le Carmen V.2 s'en écarte sur un point : il envoie Thomas en Perse et non en Inde, comme le font les Virtutes (qui réservent la Perse à Simon et Jude). La raison de cette infidélité est évidente : dans cette collection, l'Inde sert de cadre à la mission de deux apôtres. Or la poésie aime la variété. Fortunat a choisi d'assigner l'Inde à Barthélemy, si bien qu'il fallait trouver à Thomas une autre destination.

Il n'aura sans doute pas inventé cette donnée, car, bien qu'il n'existe pas, à ma connaissance, d'autre texte latin qui associe Thomas à la Perse, il en est qui l'associent aux pays des Parthes. Or la Perse de Fortunat pourrait bien n'être qu'une transposition poétique de la Parthie. La plupart des sources occidentales transmet-

65 «It is ... not certain that Venantius would not have been able to write this poem without knowledge of the narrative Virtutes apostolorum. In all cases but one, we find the same combination of apostle and missionary area elsewhere» (Rose (2013) 62). C'est exact, mais cela n'ôte rien au fait que Fortunat et les Virtutes partagent une combinaison particulière de ces données, qu'on ne retrouve nulle part ailleurs.

66 Sur les tentatives (infructueuses) de localisation de cette ville, voir Burnet (2014) 574 .

67 Rose (2013a) 63.

68 À supposer qu'une telle liste ait circulé, il serait évidemment invraisemblable qu'on ait composé les Virtutes d'après ses données ; la dépendance inverse s’impose.

69 Dans le cas de Paul, la mention de l'Illyrie pourrait être directement reprise au Nouveau Testament (Rm 15.19), mais les Virtutes apostolorum (Paul, chap. 7) restent une source possible. 
tent la tradition indienne en ce qui concerne l'activité de Thomas ${ }^{70}$. À vrai dire, les textes latins dont le poète aurait pu tirer sa localisation alternative ne sont pas légion. J'en vois quatre : l'Histoire ecclésiastique de Rufin (III.1 ; X.9), la traduction des Reconnaissances par le même Rufin (IX.29.2), Eucher de Lyon, qui reproduit les données de l'Histoire ecclésiastique (Instructiones ad Salonium I, Act. apost., 3), ou le Breviarium apostolorum. Cette dernière source est difficile à dater, mais elle pourrait fort bien être antérieure à Fortunat ${ }^{71}$. Bien qu'il soit impossible de se prononcer de façon définitive, c'est cette hypothèse que je privilégie, pour deux raisons. D'une part, contrairement aux deux autres, elle évoque les Parthes et les Mèdes à propos d'André, juxtaposition qui expliquerait au mieux la substitution de la Perse à la Parthie, puisque les Perses sont communément associés aux Mèdes. D’autre part, elle a l'avantage supplémentaire de fournir une explication possible à l'étrange mention des frimas scythes à propos de Paul. En effet, dans le Breviarium, la notice qui lui est consacrée est immédiatement suivie par celle d'André. Or cet apôtre, affirme-t-elle, praedicavit Scythiam et Achaiam. Il n'est pas exclu que ce soit une lecture inattentive de ce texte ait amené Fortunat à rapporter à Paul une donnée qui concernait en réalité l'apôtre suivant.

Si cette hypothèse s'avérait exacte, elle imposerait de placer le Breviarium au plus tard dans le troisième quart du $\mathrm{VI}^{\mathrm{e}}$ siècle. Et en ce qui concerne Fortunat, elle aurait l'intérêt de donner consistance à l'impression qu'il connaît le genre de listes d'apôtres, aussi bien sous l'angle des champs missionnaires que des lieux de sépulture - deux domaines que couvre justement le Breviarium. En effet, comme le remarque fort justement E. Rose, le contenu du Carmen VIII, 3 - et j'ajouterais pour ma part celui du Carmen V.2 - paraît supposer une certaine familiarité avec les Virtutes apostolorum, tandis que leur forme fait davantage écho aux listes d'apôtres $^{72}$.

\section{Pseudo-Venance Fortunat (?), In laudem sanctae Mariae}

Le long poème In laudem sanctae Mariae est, comme je l'ai signalé, d'authenticité contestée et semble représenter l'œuvre d'un imitateur ${ }^{73}$. Les v. 267-296 développent

\footnotetext{
70 La tradition parthe est la plus ancienne, mais elle sera supplantée par les traditions qui associent Thomas à Édesse (tombeau) et à l'Inde (champ missionnaire, souvent avec translation ultérieure à Édesse) ; sur les diverses traditions relatives à cet apôtre, voir Burnet (2014) 496-514.

71 Sur la date du Breviarium (BHL 652), voir Guignard (2019) 265 (avec davantage de références).

72 Rose (2013a) 63.

73 Voir n. 36.
} 
le thème du cortège céleste d'une façon qui rappelle le Carmen VIII.3. Les saints se rassemblent pour faire l'éloge de la Vierge Reine, qui trône aux côtés de son Fils ${ }^{74}$ :

Occurrunt proceres et gloria magna Iohannis, noscens ante Deum quam pareretur homo;

fratribus adscitis Paulo reliquisque beatis ductor apostolicus, fulgida palma Petrus.

$[\ldots]$

Hinc patriarcharum numero sociante prophetae conueniunt celso munera ferre throno,

martyrii hinc inde cohors redimita coronis, principe cum Stefano ciuica turba poli,

primitiae florum, Bethlemitica passio lactans, quae meruit nascens uiuere morte magis,

Italiae mundique caput quos Roma retentat, urbs uel Alexandri aut Antiochenus ager,

quos Hierosolymis aut quos Byzantion oris, quos Patras Ephesus Naddauer arce tenet, quos Oriens, Occasus habet, quos Africa et Arctos quosque sepultat humus, cingit et Oceanus.

Natio gens populus regiones agmina reges undique currentes ad noua dona patris, Aethiopes Thraces Arabes Daca Indus Alanus, Aurora et Vesper, Persa, Britannus adest, intrantes in regna dei pietate redempti, gaudia uisuri regis in arce poli,

quos recipit sacra porta Petri, quos ianua Pauli; postis ad Andreae limen Achaia petit.

Mattheus Aethiopes, Ephesenos ualua Iohannis, et quem quisquis amat hunc sua porta rogat.

Comme dans le Carmen VIII.3 (siderei proceres, v. 129), le poète nous fait assister à la procession des proceres (v. 267). Ces dignitaires sont, comme dans ce poème, les saints des deux testaments et les martyrs : Jean le Baptiste (v. 267), les Patriarches et les Prophètes (v. 275), les martyrs, à commencer par Étienne et les Saints Innocents (v. 277-280) et les apôtres. Cependant, contrairement à ce qu'on constatait dans le poème VIII, 3, quand bien même les martyrs chrétiens font partie du cortège, seuls des saints bibliques sont explicitement nommés ${ }^{75}$. Et sur les sept saints désignés par leur nom - Jean (le Baptiste, v. 267), Paul (v. 269 et 293), Pierre (v. 270 et 293), Étienne (v. 278), André (v. 294), Matthieu et Jean (v. 295) - cinq sont des apôtres. Les références aux apôtres se concentrent au début et à la fin du passage : au début, Pierre et

74 La royauté de la Vierge est développée aux v. 259-266 ; les éloges de la vierge occuperont les v. 315-346. Pour un commentaire de ces vers, voir Blomgren (1934) 286-294.

75 Ce n'est sans doute pas un hasard non plus que presque tous les saints désignés nominalement (concrètement tous ceux qui sont nommés ci-après, à l'exception de Matthieu) fassent partie de ceux qui occupent une place importante en Gaule au VI siècle ; cf. Beaujard (2000) 254. 
Paul ; à la fin, ces mêmes apôtres avec André, Matthieu et Jean. Cependant, la présence des apôtres parcourt de façon sous-jacente une bonne partie du passage et ce sont eux qui retiennent surtout l'attention du poète, bien plus que les autres saints.

Le thème apostolique est développé successivement de deux façons. D’abord, de façon entièrement implicite, dans la dernière partie de l'énumération des martyrs. En effet, formellement, les v. 281-286 continuent cette énumération et peuvent s'interpréter comme désignant l'ensemble de ceux qui sont ensevelis à Rome ${ }^{76}$, Alexandrie, Antioche, etc., interprétation que conforte le caractère général des v. 284 et, surtout, 285. Néanmoins, les lieux mentionnés - qui sont d'ailleurs au nombre de douze - sont presque tous susceptibles d'être reliés à tel ou tel apôtre et, en sens inverse, seul un apôtre ne peut être relié à aucun d'eux (Philippe) ${ }^{77}$. Les exemples les plus clairs sont Patras, connue comme lieu de sépulture d'André, Éphèse, comme abritant le tombeau de Jean, et, plus encore, Naddaver, toponyme qui n'est, semble-til, attesté que par la Passion de Matthieu des Virtutes apostolorum, qui y situe le tombeau de l'apôtre ${ }^{78}$. Une certaine similitude entre le v. 285 , qui fait référence aux quatre points cardinaux, et la géographie apostolique du poème V, 2 fait envisager qu'Africa (qui, dans ce contexte, pourrait avoir une portée assez générale) évoque la région où est enseveli Lebbée selon la tradition reproduite par Paulin (Lebbaeum Libyes [accepit], Natalicium 11.82). Cependant, étant donné que l'auteur s'appuie sur la Passion de Matthieu, il paraît probable qu'il suive aussi la Passion de Simon et Jude, qui est étroitement apparentée à celle de Matthieu ${ }^{79}$, comme le fait Fortunat dans le poème VIII.3, qui situe leur tombeau en Perse. Or Jude (de Jacques) est l'homologue lucanien de Thaddée-Lébbée. Autrement dit, si l'auteur suit la tradition attestée par les Virtutes apostolorum (en tout cas pour ces deux passions), il inclut sans doute Jude parmi ceux dont les tombeaux sont en Orient et ne s'occupe pas de Thaddée-Lebbée, qui ferait double emploi. Aussi est-il plus vraisemblable de lire dans Africa une référence visant particulièrement Cyprien, en écho à ce qu'on lit dans le poème VIII,.3 : Africa Cyprianum dat (v. 153). D’autant que l'Occident et le Nord (Arctos), contrairement aux onze autres régions mentionnées, n'ont aucun apôtre à fournir ${ }^{80}$. Il faut aussi relever que les cinq premières villes citées (Rome,

76 La formule Italiae mundique caput (v. 218) évoque évidemment le caput orbis du Carmen VIII.2.140.

77 Sur les raisons de cette omission, voir n. 83.

78 Cf. Lipsius (1883-1890), I, 167.

79 Voir Alibert (2005) 813 et 841.

80 Dans le poème V, 2, on l'a vu, Fortunat reliait tant bien que mal la prédication de Paul aux régions septentrionales, mais il est ici question de sépulture; même problème concernant la prédication de Philippe en Gaule (cf. Versus memoriales, v. 6 ; voir p. 386) Quant à l'Occident, la légende relative à la présence du tombeau de Jacques le Majeur en Galice n'était pas encore née. La seule échappatoire serait de supposer qu'en faisant référence à l'Occident et au Nord l'auteur penserait aux reliques apostoliques qui pouvaient se trouver dans ces régions, en particulier en Gaule, où, dès la fin du IV siècle, étaient présentes des reliques d'André, de Thomas et de Luc (Victrice de Rouen, De laude 
Alexandrie, Antioche, Jérusalem et Byzance) correspondent aux cinq patriarcats et que la volonté d'y faire référence prime sans doute ici sur la géographie apostolique. Rome ne fait évidemment aucune difficulté, Jérusalem peut revendiquer les deux Jacques (la terra beata qui les abrite selon le poème VIII.3.144 est sans doute la terre d'Israël) ou au moins l'un d'eux ${ }^{81}$, et, à défaut d'apôtre, Alexandrie a Marc. Le cas de Byzance peut se régler en prenant en compte les dépouilles apostoliques ou subapostoliques qui y ont été transférées (André, Luc et Timothée). Celui d'Antioche est plus compliqué, mais il ne serait pas impossible de lui attribuer Luc ; c'est en tout cas ce que fait une liste transmise par le Codex Fuldensis ${ }^{82}$.

Somme toute, il serait certainement excessif de faire une lecture uniquement apostolique des indications géographiques des v. 281-286, mais le fait est que les lieux nommés correspondent pour la plupart à des traditions relatives aux tombeaux des apôtres. Les deux premières colonnes du schéma ci-dessous résument les associations évidentes ou très probables (en gras) et les associations possibles ou éventuelles ; la troisième indique les équivalences (=) avec les données du Carmen VIII.3 :

\begin{tabular}{lll}
\hline Lieu & Apôtres (et martyrs) & Carmen VIII.3 \\
\hline Rome & Pierre et Paul & $=$ Aegyptus \\
\hline Alexandrie & Marc & [pas de lieu pour Luc] \\
\hline Antioche & Luc ? & $=$ terra beata \\
\hline Jérusalem & (les deux ?) Jacques & $=$ Achaïe \\
\hline Byzance & André (après transfert), Luc et Timothée (idem) $?$ \\
\hline Patras & autres martyrs ? & $=$ \\
\hline Éphèse & Jean (et Timothée [avant transfert] ?) & $=$ \\
\hline Naddaver & Matthieu & $(=)$ \\
\hline Orient & Barthélemy, Thomas, Jude et Simon & + Philippe à Hiérapolis \\
\hline Occident & autres martyrs ? & Cyprien (plutôt que Lebbée) ? \\
\hline Afrique & autres martyrs ? & \\
\hline Nord & & \\
\hline
\end{tabular}

sanctorum 6), et où, dans la seconde moitié du VI ${ }^{\mathrm{e}}$ siècle, Grégoire de Tours atteste la diffusion de reliques d'André (voir Denoël (2004) 56).

81 La plupart des listes d'apôtres anciennes assignent au moins l'un des deux Jacques à Jérusalem ; voir par ex. les trois listes mentionnées à la n. 33, ainsi que la Notita regionum (BHL 650), la liste du Codex Fuldensis (BHG 651) ou encore l’Anonyme I (voir Guignard 2015a).

82 BHL 651 ; le texte se lit par exemple dans Ranke (1868). Aucun texte de Fortunat ne nous indique où il situait le tombeau de Luc. 
La comparaison avec le Carmen VIII.3 révèle que les indications sont pour une bonne part identiques ou en tout cas compatibles, la ville indiquée par l'In laudem sanctae Mariae correspondant à la province mentionnée par le poème VIII.3. La seule différence significative résulte dans l'omission de Philippe - qui pourrait être un résultat mécanique de la volonté d'inclure les cinq patriarcats et les quatre points cardinaux sans dépasser le nombre de douze lieux ${ }^{83}$. Ce passage de l'In laudem s'appuie donc soit sur le poème VIII, $3^{84}$ soit sur les mêmes sources que lui. Il ne constitue cependant pas une simple reprise des mêmes données : d'une part, la mention de Byzance suggère que le poète relie André à la fois à cette ville et à Patras, en tenant compte du transfert de ses reliques et, peut-être, de celles de Luc et de Timothée ; d'autre part, d'autres martyrs sont manifestement pris en compte, en tout cas de façon collective, le seul que l'on puisse identifier précisément avec un haut degré de vraisemblance étant Cyprien.

Après ce premier traitement presque cryptique, le thème apostolique trouve une nouvelle expression dans la section suivante (v. 289-296), sous un autre angle : celui des régions ou peuples évangélisés. Comme l'explique Marc Reydellet, chaque nation a son protecteur apostolique qui l'accueille à une porte du ciel ${ }^{85}$. La présence des apôtres est encore implicite dans l'énumération éthnico-géographique des v. 289s., mais elle se fait explicite lorsque le poète met en scène quelques-uns d'entre eux et leur rattache des peuples ou des provinces (v. 293-296) ${ }^{86}$.

\begin{tabular}{ll}
\hline Apôtre & Peuple \\
\hline Pierre & - \\
\hline Paul & - \\
\hline André & Achaïe \\
\hline Matthieu & Éthiopiens \\
\hline Jean & Éphésiens \\
\hline
\end{tabular}

83 L'auteur devait donc inclure cinq villes ou régions (Alexandrie et Antioche, l'Occident, l'Afrique et le Nord) qui ne correspondent, semble-t-il, à aucun apôtre, tandis que Byzance et Patras se rapportent toutes deux à un même apôtre, André. Le fait que Rome et Jérusalem comptent pour deux apôtres chacune et l'Orient pour quatre compensent à hauteur de cinq apôtres au lieu des six qui auraient été nécessaires pour que la liste des Douze soit complète. Dans ces conditions, l'omission de l'un d'eux était inévitable.

84 Telle était l'opinion Blomgren (1934) 94, qui évoque une imitation de VIII.3 et peut-être aussi de V.2.

85 Reydellet (2004) 177 n. 168.

86 On remarquera que Pierre et Paul ne se voient pas assigner de peuple en particulier (les deux quos qui introduisent les relatives qui les mentionnent [v. 293] se rapportent en général aux nations nommées précédemment) ; ils apparaissent ainsi, contrairement aux autres, comme des apôtres œcuméniques. 
Le v. 296 implique que le principe s'étend aux apôtres omis : et quem quisquis amat hunc sua porta rogat. Cette indication signifie-t-elle que les peuples qui viennent d'être énumérés sont susceptibles d'être reliés à un apôtre ? Le fait que l'énumération des peuples aux v. 289s. commence par les Éthiopiens, reliés ensuite à Matthieu (v. 295), semble le suggérer, mais le poète n’a pas fourni d'autre clé. De fait, contrairement à la liste précédente, qui laissait facilement deviner des associations entre lieux, la liste de huit peuples ne s'y prête guère. Outre les Éthiopiens, seules deux relations se laissent facilement établir à la lumière du Carmen VIII.3 et des Virtutes Apostolorum : les Indiens sont à relier à Barthélemy ou (si le poète s'inspire des Virtutes sans la médiation du Carmen VIII.3) à Thomas ; la Perse, à Simon et Jude. La Thrace pourrait éventuellement être le champ de mission d'Andrée ${ }^{87}$. Quant aux quatre autres peuples (Arabes, Daces, Alains, Bretons), ils n’ont guère de liens avec les traditions apostoliques ${ }^{88}$. Le poète s'appuie-t-il sur une liste de champs de mission inconnue par ailleurs ? Le caractère plus ou moins exotique des peuples nommés suggère plutôt qu'il a tenu à insister sur l'extension de la mission apostolique en montrant qu'elle avait atteint des peuples barbares et lointains, jusqu'aux extrémités du monde connu (Éthiopiens, Indiens, Bretons ; cf. Ac 1.8). En effet, bien que les listes d'apôtres contiennent parfois des noms de peuples passablement exotiques dans telle ou telle notice, ils ne sont mis en relation qu'avec un ou deux apôtres (en particulier Thomas), tandis que le contexte invite ici à les relier plus largement à la prédication des Apôtres.

Il semble donc que, contrairement à ce qui passait pour les lieux de sépulture, le poète, tout en se souvenant du Carmen VIII.3 ou des Virtutes, n'a pas en tête une liste précise des champs de mission. Il est néanmoins probable qu'il ait une certaine connaissance de ce type de listes, puisqu'il sous-entend que différents peuples entrent par les portes de l'apôtre qui leur a annoncé l'Évangile.

Ainsi, bien que l'auteur de l'In laudem sanctae Mariae ne donne aucune liste systématique, les v. 267-296 mettent en œuvre une poétique qui joue sur deux types d'associations géographiques qu'indiquent les listes d'apôtres : champs de mission et lieux de sépulture. On rejoint la conclusion déjà atteinte à propos de Fortunat : ces deux types de liste lui sont sans doute familiers. Ce qui, dans l'hypothèse où celui-ci, n'est pas l'auteur du poème, suggère qu'il provient du même milieu.

\section{Conclusion}

$\mathrm{Au}$ terme de ce parcours, il faut d'abord souligner la rareté des énumérations (plus ou moins) complètes des membres du collège apostolique dans la poésie chrétienne

87 Cf. Grégoire de Tours, Liber de miraculis Andreae apostoli 9.

88 Le lien établi entre la Bretagne et les apôtres Matthias et Simon le Zélote par la liste grecque transmise sous le nom de Dorothée de Tyr (Schermann (1907) 156, 14 ; 157, 3), qui remonte au VIII siècle (voir Dolbeau (2012) 176s.), est tardif et isolé. 
antique. Alors que les poètes grecs ne semblent guère y avoir vu de potentiel littéraire, les Latins seront un peu plus sensibles à la poétique des listes d'apôtres. Toutefois, le recours que fera Paulin de Nole, au début du V $\mathrm{V}^{\mathrm{e}}$ siècle, à une énumération d'ampleur significative, mais (volontairement) incomplète, restera longtemps isolé. En effet, il ne trouvera un successeur que plus d'un siècle et demi plus tard en la personne de Venance Fortunat, qui donnera la première liste poétique complète dans son poème VIII.3. C'est, semble-t-il, avec lui que ce procédé s'inscrira véritablement dans la poésie chrétienne d'Occident, comme en témoignent à la fois son propre corpus (Carmen V.2 et l'In laudem sanctae Mariae, qui pourrait bien être l'œuvre d'un imitateur).

Aussi modeste soit le phénomène étudié dans ces pages, il est intéressant d'examiner cette évolution à la lumière du développement parallèle des listes et vies d'apôtres en prose. En effet, la simple énumération de noms que constituent les listes d'apôtres évangéliques n'a pas inspiré les poètes antiques ${ }^{89}$, et il semble qu'il ait fallu attendre l'existence de listes qu'on pourrait dire «à double entrée», c'est-àdire de listes reliant un apôtre et (au moins) un type donné d'information (champ de mission, lieu de mort et/ou de sépulture), pour que les poètes trouvent là une matière intéressante. En effet, Paulin, qui est le premier à l'inclure dans ses vers, a tiré ses informations d'une liste des lieux de sépulture des apôtres. Il est d'ailleurs, à notre connaissance, le plus ancien témoin d'une liste d'apôtres, genre qui était alors relativement nouveau. Fortunat s'inspirera pour sa part d'un type un peu différent de matériaux apostoliques, puisque les Virtutes apostolorum sont un ensemble de vies ou de passions des apôtres ; mais celles ne font somme toute que reproduire à l'échelle d'une collection le principe des listes. En outre, il connaît très vraisemblablement au moins une liste d'apôtres, peut-être le Breviarium apostolorum. Quelle que soit exactement sa source, la récurrence d'énumérations des apôtres ou de certains d'entre eux en lien avec leurs lieux de mission ou de sépulture - deux thèmes couverts par les listes - suggère que Fortunat et, s'il est différent, l'auteur du In laudem sanctae Mariae, avaient une certaine connaissance du genre.

Ce rapport a priori improbable entre listes d'apôtres et inspiration poétique aboutir à la production d'un type nouveau de poèmes, qui sont entièrement consacrés aux champs de mission ou aux tombeaux des apôtres et qui, à ce titre, représentent le pendant exact des listes d'apôtres en prose. Le premier exemple pourrait en être un bref poème sur les régions évangélisées par les apôtres auquel Schermann a donné le titre de Versus memoriales $\left(\mathrm{BHL}^{\mathrm{n}} 654 \mathrm{k}\right)^{90}$, si tant est que, comme on l'a proposé, cette pièce ne soit pas antérieure à la fin du $\mathrm{VI}^{\mathrm{e}}$ siècle $^{91}$ :

Romanis Petrus, Andreas sanctus Achiuis,

Iacobus Hispanis, Asiae dat iura Iohannes.

89 À l'exception de Grégoire de Nazianze ; voir n. 7.

90 Voir mon étude et mon édition : Guignard (2019).

91 Quicherat (1840-1841) 147. 
Aethiopes Matthaeus adit, loca Parthica Thomas.

Nilicolas Symon, Hebraeos Matthia cogit.

Alter habet Solymam Iacobus, Iuda Mesopotamos.

Bartholomeus Indos, Gallos tum porro Philippus.

Paulus adit gentes totus quas continet orbis.

En tout état de cause, ces quelques vers sont un excellent exemple d'une veine poétique que les poètes médiévaux, qu'ils s'expriment en latin ou dans des langues vernaculaires, continueront à exploiter ${ }^{92}$.

\section{Bibliographie}

Alibert (2005): Dominique Alibert - Gisèle Besson - Michèle Brossard-Dandré - Simon C.

Mimouni, «Actes latins des apôtres», in: P. Geoltrain - J.-D. Kaestli (éd.), Écrits apocryphes chrétiens, vol. 2 (Bibliothèque de la Pléiade 516), Paris, Gallimard, 735-864.

Beaujard (2000): Brigitte Beaujard, Le Culte des saints en Gaule. Les premiers temps. D’Hilaire de Poitiers à la fin du Vle siècle, Paris, Cerf.

Blomgren (1934): S. Blomgren, Studia fortunatiana, 2 (Uppsala Universitets årsskrift, 1934, 2), Uppsala, Almqvist \& Wiksell.

Bovini (1977): Giuseppe Bovini, Il Mausoleo di Teodorico, Ravenna.

Campanale (1980): Maria I. Campanale, «ll De Virginitate di Venanzio Fortunato (carm. 8,3 Leo): un epitalamio mistico», in: Invigilata Lucernis 2, 75-128.

Cattaneo (2001): Enrico Cattaneo, «La Devozione ai santi nei carmi di san Paolino di Nola», in: La Civiltà Cattolica 3615, 266-276.

Ceccarelli (2015): Lucio Ceccarelli, «Note sul Carmen in Laudem Sanctae Mariae attribuito a Venanzio Fortunato: metro e autenticità», in: Vichiana. Rassegna di studi filologici e storici 52/1, 87-102

Charlet (1985): Jean Louis Charlet, «L'inspiration et la forme bibliques dans la poésie latine chrétienne du $\mathrm{II}^{\mathrm{e}}$ au $\mathrm{VI} \mathrm{e}^{\mathrm{e}}$ siècle», in: J. Fontaine - Ch. Pietri (dir.), Le monde latin antique et la Bible (Bible de tous les temps 2), Paris, Beauchesne, 613-643.

Cutino (2005): Michele Cutino, «L'epopea taumaturgica di S. Felice nei Carmi 26 e 19 di Paolino di Nola», in: Auctores nostri 2, 47-80.

Dijkstra (2016): Roald Dijkstra, The Apostles in Early Christian Art and Poetry, Leiden (Supplements to Vigiliae Christianae 134).

Doignon (1961): Jean Doignon, «'Procer', titre donné à Saint Martin dans une inscription gallo-romaine de Vienne», in: N.N. (éd.), Saint Martin et son temps. Mémorial du XVle

92 Voir BHL 654 ; BHL $^{\mathrm{s}}$ 654a, 654b, 654f ; BHL $^{\mathrm{n}} 654 \mathrm{~g}, 654 \mathrm{~h}$; on peut ajouter en tout cas l'hymne $O$ Dei verbum (éd. J. Castro Sánchez, Hymnodia hispanica [Corpus Christianorum. Series latina 167], Turnhout, Brepols, 2010, p. 479-484). Dans le domaine vernaculaire, il n'existe à ma connaissance aucun catalogue de textes. Je ne suis en mesure de citer que les textes suivants : en vieil irlandais, un poème auquel son éditeur a donné le titre d'Abstammung der zwölf Apostel (Meyer (1912) 107 ; inc. Petor co treib Iuda áin) ; en vieil anglais, Cynewulf (IX siècle ?), The Fates of the Apostles ; en vieil allemand, la strophe V de la Chanson d'Annon, évêque de Cologne (†1075) ; en vieil islandais, Petrs saga postola, ch. 85 (Unger 1874). Je ne serais pas surpris qu'une recherche systématique en fasse émerger un nombre bien plus considérable. 
centenaire des débuts du monachisme en Gaule, 361-1961 (Studia Anselmiana 46), Romae, Orbis catholicus, 151-158.

Dolbeau (2012): François Dolbeau, Prophètes, apôtres et disciples dans les traditions chrétiennes d'Occident (Subsidia Hagiographica 92), Bruxelles : Société des Bollandistes.

Duchesne (1895): Louis Duchesne, "Les anciens recueils de légendes apostoliques », in: N.N. (éd.), Compte rendu du troisième Congrès scientifique international des catholiques tenu à Bruxelles du 3 au 8 septembre 1894, Bruxelles, Société belge de librairie, vol. 5, 67-79.

Guttilla (2006): Giuseppe Guttilla, «Il De errore profanarum religionum di Firmico Materno nel Carm. 19 di Paolo di Nola», in: Revue des études augustiniennes et patristiques 52/2, $355-377$.

Evenepoel (2016): Willy Evenepoel, Studies in the Christian Latin Poetry of Late Antiquity

(Spicilegium sacrum Lovaniense. Études et documents 53), Leuven, Peeters, 399-414.

Fux (2003): P.-Y. Fux, «Les patries des martyrs : doctrines et métaphores chez quatre poètes de l'Antiquité tardive», in: Connaissance des Pères de l'Église 92, 52-60.

Guignard (2015a): Christophe Guignard, «La tradition grecque de la liste d'apôtres »Anonyme l« (BHG 153c), avec un appendice sur la liste BHG 152n», in: Apocrypha 26, 171-209.

Guignard (2015b): Christophe Ch. Guignard, «Greek Lists of the Apostles: New Findings and Open Questions», in: Zeitschrift für Antikes Christentum - Journal of Ancient Christianity 20/3 (2016), p. 469-495.

Guignard (2019): Christophe Guignard, «Un bref poème latin sur les champs de mission des apôtres (»Versus memoriales«, BHLn 654k)», in: Revue bénédictine 129, 254-266.

Joye (2008): Sylvie Joye, «Agnès (après 500-vers 587)», in: Dictionnaire des femmes de l'Ancien Régime, [Paris], Société internationale pour l'étude des femmes de l'Ancien Régime (SIEFAR).

Labande-Mailfert (1986): Yvonne Labande-Mailfert, "Les débuts de Sainte-Croix», in: E.-R. Labande (dir.), Histoire de l'Abbaye Sainte-Croix de Poitiers (Mémoires de la Société des antiquaires de l'Ouest, $4^{\mathrm{e}}$ sér., 19), Poitiers, Société des antiquaires de l'Ouest.

Lipsius (1883-1890): Richard Adelbert Lipsius, Die apokryphen Apostelgeschichten und Apostellegenden. Ein Beitrag zur altchristlichen Literaturgeschichte, 3 vol. et 1 vol. suppl., Braunschweig, C. A. Schwetschke.

Luongo (2002 - 2006): Gennaro Luongo, «La Geografia della Santità nel Carme XIX di Paolino di Nola», in: Impegno e Dialogo 15, 167-191.

Luongo (2006): Gennaro Luongo, «Martyr stella loci (Paul. Nol., carm. 19, 15)», in: Auctores nostri 4, 373-396,

Meyer (1912): Kuno Meyer, «Mitteilungen aus irischen Handschriften »:, in: Zeitschrift für celtische Philologie 8, 108-112.

Nisard (1887): Charles Nisard, Venance Fortunat. Poésies mêlées, Paris, Firmin-Didot.

Piras (2001): Antonio Piras, «Roma Petro Pauloque potens: Roma e gli apostoli negli scritti di Paolino di Nola», in: N.N. (éd.), Pietro e Paolo. Il loro rapporto con Roma nelle testimonianze antiche. XXIX Incontro di studiosi dell'Antichità cristiana, Roma, 4-6 maggio 2000 (Studia Ephemeridis Augustinianum 74), Roma, Institutum Patristicum Augustinianum, 2001, $277-283$.

Quicherat (1840-1841): Jean Quicherat, «Fragments inédits de littérature latine», in: Bibliothèque de l'École des Chartes 2, 115-147.

Ranke (1868): Ernestus Ranke, Codex Fuldensis. Novum Testamentum Latine interprete Hieronymo ex manuscripto Victoris Capuani, Marburgi, N.G. Elwerti.

Reydellet (1998): Marc Reydellet, Venance Fortunat. Poèmes, t. 2 : Livres V - VIII [Collection des universités de France. Série latine 374], Paris, Les Belles Lettres.

Reydellet (2004): Marc Reydellet, Venance Fortunat. Poèmes, t. 3 : Livres IX - XI. Appendice - In laudem sanctae Mariae [Collection des universités de France. Série latine 374], Paris, Les Belles Lettres. 
Roberts (1989): Michael Roberts, The Jeweled Style: Poetry and Poetics in Late Antiquity, Ithaca.

Roberts (2009): Michael Roberts, The Humblest Sparrow, The Poetry of Venantius Fortunatus, Ann Arbor : University of Michigan Press.

Rose (2012): Els Rose, «Virtutes Apostolorum: Editorial Problems and Principles», in: Apocrypha 23, 11- 45 .

Rose (2013a): Els Rose, «Virtutes apostolorum: Origin, Aim and Use», in: Traditio 68, 57-96. Rose (2013b): Els Rose, «Abdias scriptor vitarum sanctorum apostolorum? The "Collection of Pseudo-Abdias « reconsidered», Revue d'histoire des textes n.s. 8, 227-268.

Williams (1978): Gordon Williams, Change and Decline. Roman Literature in the Early Empire (Sather Classical Lectures 45), Berkeley, University of California Press.

Schermann (1907): Theodor Schermann, Prophetarum vitae fabulosae, indices apostolorum discipulorumque Domini, Dorotheo, Epiphanio, Hippolyto aliisque vindicata (Bibliotheca scriptorum Graecorum et Romanorum Teubneriana), Lipsiae, B. G. Teubner).

Starowieyski (1996): Marek Starowieyski, «La légende de saint Jacques le Majeur», in: Apocrypha 7, $193-203$.

Trout (1999): Denis E. Trout, Paulinus of Nola. Life, Letters, and Poems, Berkeley, University of California Press.

Unger (1874): Carl R. Unger, Postola Sögur. Legendariske fortälliger om apostlernes liv, Christiania, B.M. Bentzen.

Vinogradov (2001): Andrey Vinogradov, «Апостольские списки [Listes d’apôtres]», in: Православная энциклопедия [Encyclopédie orthodoxe], t. 3, 121-124. 